\title{
Association of salivary-assessed oxytocin and cortisol levels with time of night and sleep stage
}

\author{
Mark Blagrove $\cdot$ Nathalie C. Fouquet • Alison L. Baird · Edward F. Pace-Schott • \\ Anna C. Davies · Jennifer L. Neuschaffer · Josephine A. Henley-Einion • \\ Christoph T. Weidemann $\cdot$ Johannes Thome $\cdot$ Patrick McNamara $\cdot$ Oliver H. Turnbull
}

Received: 15 March 2012/Accepted: 25 July 2012/Published online: 22 August 2012

(C) Springer-Verlag 2012

\begin{abstract}
There have been proposals for REM to have a function of emotional memory consolidation, and also for REM sleep to be involved in the promotion of attachment behaviour. The hormones cortisol and oxytocin, respectively, may be involved in these proposed REM sleep functions. However, there are conflicting reports on whether levels of cortisol differ between sleep stages when time since sleep onset (SSO) is controlled, and virtually no literature on whether levels of oxytocin differ between sleep stages. This study thus investigated the changes in levels of oxytocin (OT) and cortisol (CT) across the night, and whether these levels differ between REM and N2 sleep when time SSO is controlled. 20 participants (10 males, 10 females, mean age $=20.45, \mathrm{SD}=2.01$ ) were awakened 10 min into REM and N2 sleep periods in the sleep
\end{abstract}

M. Blagrove $(\varangle) \cdot$ N. C. Fouquet · A. C. Davies ·

J. L. Neuschaffer · J. A. Henley-Einion .

C. T. Weidemann $\cdot$ J. Thome

Department of Psychology, College of Human and Health

Sciences, Swansea University, Swansea SA2 8PP, UK

e-mail: m.t.blagrove@swansea.ac.uk

A. L. Baird

Institute of Psychiatry, Kings College London, London, UK

E. F. Pace-Schott

Massachusetts General Hospital, Harvard Medical School,

Boston, USA

J. Thome

Department of Psychiatry, Rostock University, Rostock,

Germany

P. McNamara

Boston University, Boston, USA

O. H. Turnbull

Bangor University, Bangor, UK laboratory and gave saliva samples which were assayed for OT and CT. Levels of OT were relatively constant across the night, whereas CT increased significantly. REM and N2 did not differ significantly neither for OT nor for CT. The study has implications for models of sleep-dependent memory consolidation that incorporate the late sleep increase in cortisol as a functional component of memory consolidation, and also for the medical diagnostic assaying of OT during sleep.

Keyword Sleep · REM sleep · N2 sleep · Oxytocin · Cortisol - Emotional attachment - Behavioural attachment . Memory consolidation

\section{Introduction}

This study addresses whether levels of oxytocin and cortisol differ between REM sleep and stage 2 sleep (N2). The wider theoretical background to the study follows from proposals for possible functions of REM sleep. Although there is the null hypothesis that REM is a "superfluous" default state (Horne 2000), the current work derives from two theories of a function for REM sleep, that it is involved in emotional memory consolidation and in the promotion of attachment.

Whereas there is considerable evidence that REM sleep is involved in procedural memory consolidation and NREM sleep in declarative learning (Gais and Born 2004; Smith 2010), it is REM sleep that appears to be involved in emotional declarative memory consolidation (Wagner et al. 2001). There have been proposals that the increased cortisol in the second part of the night is a functional component of emotional memory consolidation during REM sleep (Payne and Nadel 2004; Wagner and Born 2008; 
Payne 2010). We thus aimed to compare cortisol (CT) levels following awakenings from REM sleep with those following N2 sleep, hypothesising that REM sleep CT would be higher than N2 CT. A second proposal for a function of REM sleep is that it is involved in promoting attachment behaviour (McNamara et al. 2001). This proposal is based on evidence of commonalities in brain physiology between areas of the brain activated during REM sleep and those areas involved in attachment. The review by Nielsen and Lara-Carrasco (2007) on an emotion-regulation function for sleep, following interactions and behaviour with other people while awake, provides support for this proposal. As oxytocin (OT) is involved with attachment processes and social behaviour, it was hypothesised that OT release is higher during REM than during NREM sleep. Contrary to this hypothesis, however, is the argument that, as the posterior pituitary is presumed to be the primary source of salivary OT (Carter et al. 2007), and as REM sleep in humans begins during decreased secretory activity of the anterior pituitary (Follenius et al. 1988), it might be expected that OT release is lower during REM than during NREM sleep (although there are differences in secretory processes between anterior and posterior hypothalamus).

Aside from the theoretical issue of REM sleep function, there are further issues of the involvement of OT and CT with medical disorders of sleep that give a practical reason to study the timecourse of these hormones across the night. As OT has anxiolytic and sedating effects, and important connections in the paraventricular nucleus of the hypothalamus (which has connections to various areas that are critical for regulating sleep and arousal), OT may have a role in regulating sleep-wake behaviour. Furthermore, as OT is involved in promoting affiliative behaviours, Troxel (2010) suggests that the possible role of OT in human co-sleeping relationships may be an important area of investigation. She also suggests that oxytocin may have an etiological role in the development of psychiatric disorders, including depression, anxiety, and autism spectrum disorders, which are characterized by sleep disturbances (Hollander et al. 2003; Panksepp 2006). Cortisol has been proposed as implicated in other sleep disorders, such as obstructive sleep apnea (Tomfohr et al. 2012) and insomnia (Bonnet and Arand 2010). These biomarkers may thus be important for investigating the etiology and maintenance of these sleep disorders.

\section{Oxytocin}

Oxytocin (OT) is a hormone associated with attachment that is produced by neurons of the hypothalamic paraventricular nucleus ( $\mathrm{PVN}$ ) projecting to the posterior pituitary, from where OT is secreted into the peripheral circulation
(Gimpl and Fahrenholz 2001). Whereas OT's peripheral roles in parturition and lactation are well known, extensive central projections also exist from oxytocinergic neurons to limbic and autonomic structures that subserve its role in emotional attachment, positive physical contact with a partner, and social cognitive processes (reviewed in Bartz and Hollander 2006; Heinrichs et al. 2009; Campbell 2010; Gordon et al. 2011; Meyer-Lindenberg et al. 2011). Regarding the relationship between OT and sleep, intracerebral OT modulates sleep-wake behaviour in male rats (Lancel et al. 2003), and under basal, stress-free conditions, endogenous OT may promote sleep. However, Lancel et al. (2003) propose that, conversely, the high brain levels of OT after central OT infusion may produce a condition of stress accompanied by behavioural arousal and increased vigilance, leading to reduced sleep and increased sleep onset latency.

There is a brief literature on OT release during sleep in humans. Forsling et al. (Forsling et al. 1998; also reported in Forsling 1993) studied healthy male subjects in the age range $22-40$ years $(M=25$ years). Serum OT between 0200 and 0400 hours was found to have a level over twice that at 1700 to 0000 hours, but with a considerable variation between participants, in terms of both the timing and magnitude of the peak, and with 5 of the 15 participants showing two peaks. In contrast, Amico et al. (1983) measured OT in plasma hourly for a 24-h period from six healthy men and six healthy women and found no diurnal variation of OT. Similarly, Parker et al. (2010) took blood samples through the night and found no peak in peripheral OT in normal participants, although there was a peak at 0330 hours for depressed participants, who also exhibited a higher level of OT during the night than did normal controls.

The literature is, thus, not clear regarding circadian or sleep effects on OT. We considered that if there are variations in OT during sleep, then this may be due to modulation by sleep stage. To our knowledge the only published result regarding sleep stage and OT is one sentence in an abstract by Zetzsche et al. (1996), stating that there is no interaction between serum OT and sleep stage. Following the proposal by McNamara et al. (2001) on REM sleep and attachment, described above, we aimed to produce a more substantial report on sleep stage and OT, hypothesising that OT is higher during REM than NREM sleep.

\section{Cortisol}

Cortisol (CT) is a hormone associated with stress. It is the adrenal cortical output hormone of the hypothalamic-pituitary-adrenal axis that, along with the more rapid sympathetic activation and epinephrine release, constitutes the mammalian stress response (Ulrich-Lai and Herman 2009). Plasma and salivary CT show a clear circadian variation with a peak occurring in the early morning, steady decline 
across the day (in the absence of acute stressors), and with a minimum occurring during late-evening waking and early sleep (Davidson et al. 1991; Gronfier and Brandenberger 1998; Pannain and Van Cauter 2007; Van Cauter and Tasali 2011). CT secretion is thus primarily under circadian control. It is unclear whether this circadian CT secretion is modulated by sleep stage. There are indications that onset of REM episodes may be accompanied by decreased secretion of CT (Born et al. 1986; Follenius et al. 1992; Gronfier et al. 1999), although this was not found by Bierwolf et al. (1997), and Follenius et al. (1992) conclude that CT increases do not seem to be related to any specific sleep stage. Furthermore, a lack of relationship between sleep stage at awakening and cortisol awakening response was found by Elder et al. (2012). In contrast, Gronfier et al. (1997) found a positive relationship between CT secretion during sleep and theta band power, which is characteristic of REM, N1 and N2 sleep.

As there is an inconsistent empirical literature, and a proposal described above that CT has a role in REM sleepdependent memory consolidation, we thus wished to investigate whether CT in REM sleep exceeds CT in NREM sleep, when time since sleep onset (SSO) is controlled. N2 was used as a comparison condition, because, unlike NREM stage 3 [N3, or slow wave sleep (SWS)], it is abundant throughout the night. In contrast, N3 episodes predominate during the first half of the night and have been related to an additional decrease in cortisol beyond the circadian nadir occurring at this time of night (Follenius et al. 1992; Gronfier et al. 1997; Pannain and Van Cauter 2007; Van Cauter and Tasali 2011).

To summarise, this study seeks to investigate the time course of OT and CT levels across the night, and to investigate whether there are differences in levels of OT and in levels of CT between REM and N2 sleep when controlling for time SSO. To undertake these investigations, saliva sampling for OT and CT was used. Given the proposal that REM sleep has a function of promoting attachment, and the proposal that cortisol has a functional interaction with REM sleep to promote memory consolidation, we hypothesised that REM sleep would have a higher level of OT, and a higher level of CT, than N2 sleep.

\section{Method}

Participants: 20 participants (10 males, 10 females, mean age $=20.45$, $\mathrm{SD}=2.01$ ) were recruited. All were students. They were screened so as to include only good sleepers, without extremes of usual bedtime, and without recent or current illness or medical problems. All female participants took the dual-hormone contraceptive pill. Apart from the contraceptive pill, participants were not taking any prescription medications, including medications that are psychoactive, or affect sleep, or affect endocrine function. All participants gave written informed consent to taking part: the study was approved by the Swansea University Department of Psychology Research Ethics Committee.

Pre-sleep self-report testing for anxiety and stress

All participants completed the Profile of Mood States Bipolar version (POMS BI; Lorr 1984), which enabled assessment of the composed-anxious dimension (higher scores indicate composed, scores vary -18 to +18$)$, and the General Health Questionnaire, 28 item version (GHQ 28; Goldberg and Williams 1991), to assess medical, health and stress complaints (scores vary 0-84).

Sleep laboratory, instrumental awakening and saliva-collection procedures

Participants slept in the sleep laboratory for two nights, either on a Monday and Wednesday or on a Tuesday and Thursday, the two nights being in the same week. Sleep was monitored by polysomnography with electrodes at F4 and $\mathrm{C} 4$ for EEG, above right outer canthus and below left outer canthus for EOG-detected eye movements, on the left and right mastoids for reference, and on the chin for electromyography (EMG). Sleep scoring followed the AASM Manual for the Scoring of Sleep (Iber et al. 2007, for the American Academy of Sleep Medicine).

Awakenings were not scheduled to occur during the first 80 min of sleep. The first awakening was scheduled from the first $\mathrm{N} 2$ period after the first $80 \mathrm{~min}$ of sleep; awakening occurred after $10 \mathrm{~min}$ of $\mathrm{N} 2$, then at the end of 10 min of the next REM period, and thereafter, whenever 10 min of either REM or N2 were obtained. Participants produced a saliva sample during the period 3-8 min after awakening by expectorating down a straw into a test tube (SaliCap, IBL, Hamburg, Germany). Participants could chew on a piece of tasteless Parafilm (American National CAL, Chicago, IL, USA) if needed.

Sleep laboratory schedules

Participants arrived in the sleep laboratory at 2200 hours. They were wired up for polysomnography, and gave three saliva samples at 30 -min intervals. At $\sim 2400$ hours, they prepared for bed. After the first $80 \mathrm{~min}$ of sleep, and until final awakening, saliva was sampled $10 \mathrm{~min}$ into REM and N2 sleep. Saliva was then sampled three times after final awakening in the morning. In the laboratory, before and during sleep, participants did not eat or drink. In the morning, participants did not have food or drink during the $30 \mathrm{~min}$ before each post-sleep saliva sampling. 
Saliva sampling for OT

Although saliva sampling for CT is a standard and longstanding procedure, recent advances have enabled the assessment of the far smaller amounts of OT in saliva (Carter et al. 2007; White-Traut et al. 2009). This work has detected changes in salivary OT in individuals receiving massage (Carter et al. 2007) and in breastfeeding mothers (Carter et al. 2007; White-Traut et al. 2009). The methodological advance has been to dry (in the cold) and resuspend all samples, so as to concentrate samples fourfold and thus produce levels within the range of the enzymelinked immunosorbent assay (ELISA). The success of the saliva concentration method is shown in that salivary and serum OT correlate significantly with $r=0.59$ (Grewen et al. 2010).

As this study aims to detect any association between sleep stage and OT and CT levels, consideration was given to the length of time the participant would be in a sleep stage before awakening. Gordis et al. (2008) used a serial subtraction task as a stressor and found that salivary CT peaked at $10 \mathrm{~min}$ post-stressor and returned to baseline levels at $20 \mathrm{~min}$ post-stressor. Peak response of salivary CT to the Trier Social Stress Test also occurs 10 min posttest (Gordis et al. 2006). For OT, Grewen et al. (2010) found a significant salivary OT response to a 5-min period of mother-child interaction when assessed at the end of a further 7-min post-interaction period. Given these poststimulus delays in salivary CT and OT responses, and balancing this with the need to capture a sleep stage before it changes to another sleep stage, we scheduled awakenings at 10 min into REM and N2 sleep with saliva collection starting at approximately 3 min after awakening.

\section{Enzyme immunoassay method}

The method of White-Traut et al. (2009) and Carter et al. (2007) was followed. Each sample was given a random identifying number. Samples were frozen and stored at $-20^{\circ} \mathrm{C}$. Each sample was divided for OT and CT assaying. Salivary OT and CT were both assayed using commercially available ELISAs. Samples of OT and CT were tested in duplicate. CT was measured using an ELISA kit produced by IBL International (Hamburg, Germany). IBL International report a $30 \%$ cross-reactivity for prednisolone, a cross-reactivity for all other analytes of $<10 \%$, and a minimum detection limit of $0.005 \mu \mathrm{g} / \mathrm{dl}$. OT was measured using an ELISA kit developed by Assay Designs, Inc. (Ann Arbor, MI, USA). Assay Designs report a low cross-reactivity for similar neuropeptides found in mammalian sera at $<0.001 \%$ and a minimum detection limit of $4.68 \mathrm{pg} / \mathrm{ml}$. To allow measurement of salivary OT using a reliable portion of the standard curve, samples were concentrated prior to assay. Using a Lypholizer, $1 \mathrm{ml}$ of supernatant from each sample was freeze-dried at $-70{ }^{\circ} \mathrm{C}$ by vacuum centrifugation and then reconstituted in $250 \mu$ l of assay buffer, resulting in a sample with a salivary concentration four times higher than the original.

\section{Statistical analyses}

Sex differences in OT and CT were investigated by independent samples Mann-Whitney tests. Participants provided different numbers of samples and at various awakening times. To enable within-subjects analyses of the levels of OT and CT across the night, the data were combined into timeperiod bins, so as to increase the number of participants providing data for all bins in a series of combined time periods. Awakenings were classified into early-sleep, middle-sleep and late-sleep bins. Levels of OT and CT for presleep, early sleep, middle sleep, late sleep and post-sleep were compared by Friedman's test: this ranking procedure was used due to large individual differences in mean levels of OT and CT. If a participant had more than one sample in a bin, then the mean of the samples was used, and the participant would only be included in the analysis if they had at least one sample in each of the bins. Each participant thus contributed, at most, only one value to each bin, and all participants who were included in that analysis thus contributed equally to each (OT and CT) analysis. The Friedman's tests were also rerun using hot deck imputation so as to include three participants who had some missing data from some of the time bins.

To compare levels of OT or CT between sleep stage (REM and N2) while controlling time of night, Wilcoxon tests were conducted between REM and N2 for OT/CT at each of the time periods (early, middle and late sleep). A more fine-grained analysis to compare levels of OT and CT after REM and N2 awakenings was then conducted using time SSO in an ANCOVA, as levels of OT and CT in REM and also in $\mathrm{N} 2$ were approximately normally distributed.

\section{Results}

There were 76 REM and 66 N2 awakenings. Awakenings occurred from 1.34 to $9.10 \mathrm{~h} \mathrm{SSO}$. The numbers of analysable OT samples were: 98 pre-sleep; 62 REM; 54 N2; 49 post-sleep. The numbers of analysable CT samples were: 104 pre-sleep; 69 REM; 61 N2; 54 post-sleep.

There were no significant differences between males and females neither for pre- or post-sleep OT nor for OT levels in early, middle or late sleep (all Mann-Whitney $|z s|<0.6$ ). Males had higher CT levels than females for all time bins but none of these differences was significant (MannWhitney test $z$ s varied from 0.36 to 1.66 ). 


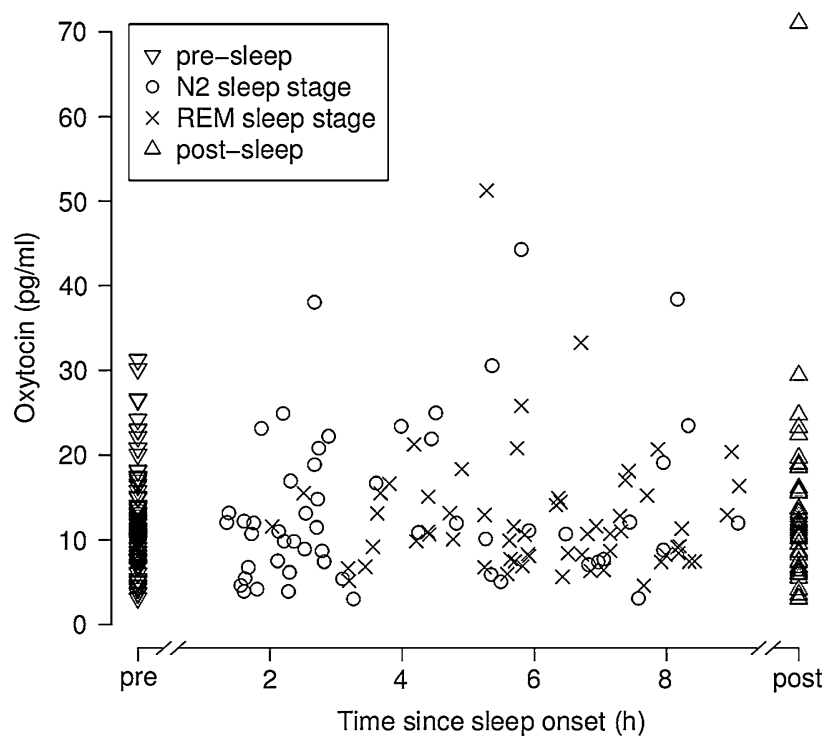

Fig. 1 Oxytocin as a function of time since sleep onset and sleep stage (263 samples, 20 participants)

For the 20 participants, POMS mean composed/anxious score $=4.5(\mathrm{SD}=6.5, \min =-10, \max =+13)$; mean GHQ score $=18.0(\mathrm{SD}=9.4, \min =8, \max =46)$. Postsleep CT correlated at Spearman's $\rho=-0.46$ with composed/ anxious, and $\rho=0.40$ with GHQ, whereas pre-sleep CT correlated at $\rho=0.02$ with GHQ and at $\rho=0.02$ with composed/ anxious. Following corrections for multiple correlations the correlations with post-sleep CT were not significant.

OT and CT levels across the night combining REM and N2 awakenings

Figure 1 shows the values of OT from all 263 OT samples collected from 20 participants as a function of time SSO and sleep stage.

Figure 2 shows the values of CT from all 288 CT samples collected from 20 participants as a function of time SSO and sleep stage.

To enable within-subjects analyses of the levels of OT and $\mathrm{CT}$ across the night, awakenings were classed as early sleep ( $<4 \mathrm{~h} \mathrm{SSO}$ ), middle sleep (4-6.6 h SSO) and late sleep ( $>6.6 \mathrm{~h} \mathrm{SSO}$ ) by dividing awakening times into three approximately equal-sized groups (early sleep, $34 \%$ of awakenings; middle sleep $33 \%$ of awakenings; late sleep, $33 \%$ of awakenings). These three periods were also of similar length: early sleep, 1.34-3.99 h SSO; middle sleep, 4.03-6.59 h SSO; late sleep, 6.61-9.10 h SSO.

\section{Oxytocin}

Seventeen participants provided OT data for each of the combined sleep periods, and for pre- and post-sleep. Figure 3 shows the mean OT data from these 17 participants.

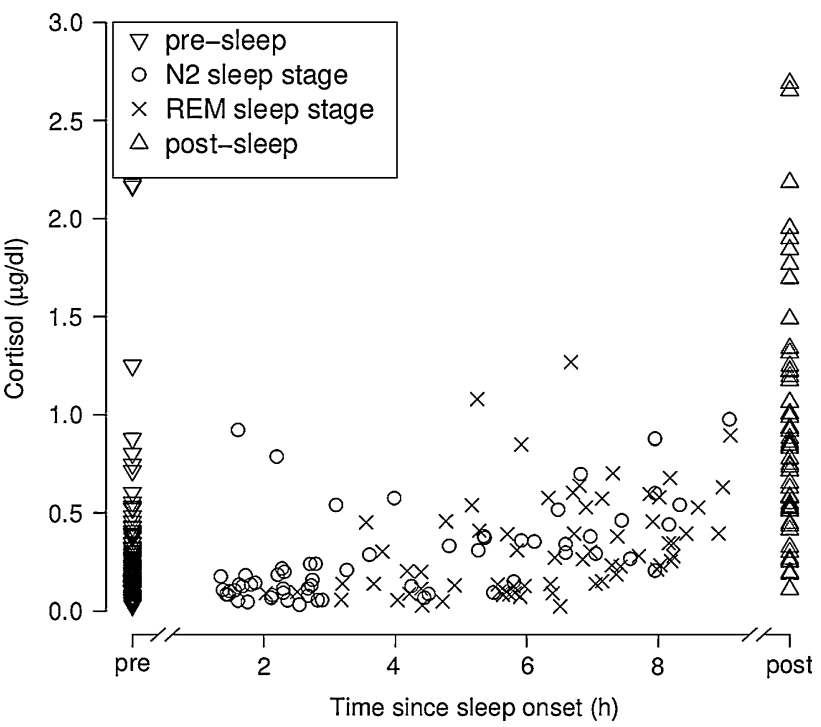

Fig. 2 Cortisol as a function of time since sleep onset and sleep stage (288 samples, 20 participants)

A Friedman test on these data found that the levels of OT did not differ significantly between the time periods $\left(\chi^{2}(4)=3.06, p=0.548\right)$. The Friedman test was rerun after having used hot deck imputation so as to include the three participants with missing data for some of the time bins. We substituted the data from cells of participants with a full data set, for the missing data for each of the excluded three participants. Substituted data were taken from the participant who had data that were the most similar to the partial data of the previously excluded participant. The Friedman test results using hot desk imputation were negligibly different from the results above $\left(\chi^{2}(4)=2.44\right.$, $p=0.655, n=20$ ).

\section{Cortisol}

Seventeen participants provided CT data for each of the combined sleep periods, and for pre- and post-sleep.

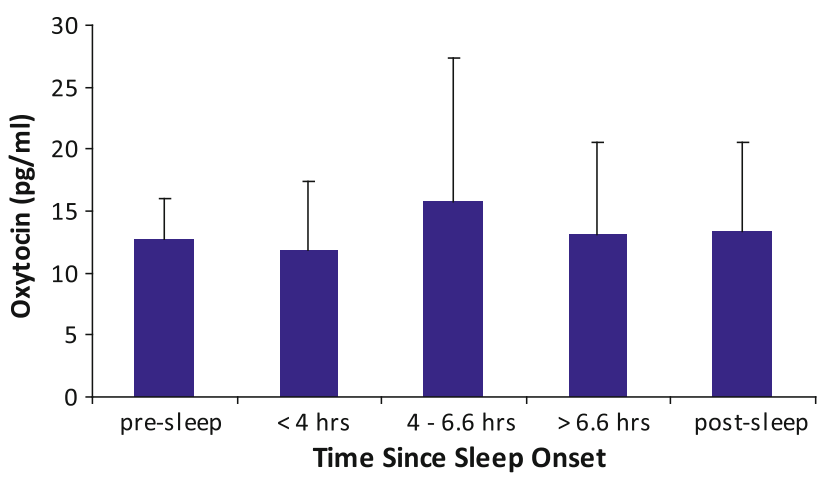

Fig. 3 Levels of salivary oxytocin before, during and after sleep $(n=17)$. Error bars are standard deviations 


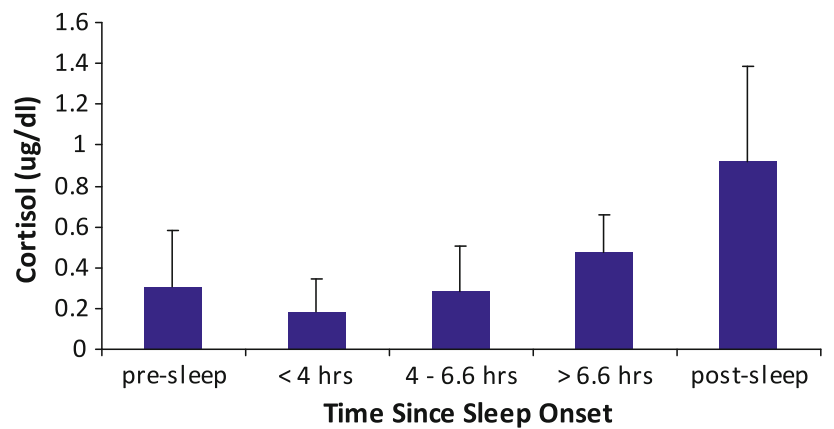

Fig. 4 Levels of salivary cortisol before, during and after sleep $(n=17)$. Error bars are standard deviations

Figure 4 shows the mean CT data from these 17 participants, indicating an increase in CT across the night, and after awakening.

A Friedman test on these data found that the levels of CT differed significantly between the time periods $\left(\chi^{2}(4)=38.49, p<0.001, n=17\right.$; results using hot desk imputation were negligibly different from these results, $\left.\chi^{2}(4)=46.80, p<0.001, n=20\right)$. Due to the number of significant comparisons the Wilcoxon test results for the comparisons in Fig. 4 are represented in the following table (Table 1) rather than in the figure.

\section{Comparisons between REM and N2 sleep for OT and CT levels}

Table 2 shows the levels of OT and CT for REM and N2 at each of the sleep periods (early, middle and late sleep). Wilcoxon tests were conducted between REM and N2 awakenings for OT and CT at each of the time periods. There were no significant differences in OT between REM and N2 for any time period (all $|z \mathrm{~s}|<0.6$ ), and no significant differences in CT between REM and N2 for any time period (all $|z \mathrm{~s}|<1.5$ ). Note that for each sampling period, some participants did not provide a sample from both REM and N2, and so the number of participants in each comparison is less than the total number of participants.

A more fine-grained analysis of the individual OT and CT levels across all awakenings was then conducted, using the data from all 20 participants. In Fig. 1, it is apparent that there was considerable overlap in the distributions of OT levels for the two sleep stages under consideration. To statistically confirm that OT levels did not vary significantly with sleep stage, we performed an ANCOVA on the OT levels with sleep stage as a factor and time SSO as a covariate (because of unequal sampling we used Type I sum of squares and included time SSO in the model first to remove any influence of the covariate on our main effect). As expected from Fig. 1 and the above analyses, the effect of time SSO was not significant $(F(1,108)=0.76$,
MSE $\left.=68.93, p=0.386, \eta_{p}^{2}=0.01\right)$ and neither was that of sleep stage $(F(1,108)=1.75, \quad \mathrm{MSE}=68.93$, $\left.p=0.188, \quad \eta_{p}^{2}=0.02\right)$ nor that of the interaction $\left(F(1,108)=0.64, \mathrm{MSE}=68.93, p=0.427, \eta_{p}^{2}=0.01\right)$.

We carried out a corresponding analysis with the CT levels presented in Fig. 2 as the dependent variable and found a significant effect for time $\operatorname{SSO}(F(1,122)=21.46$, MSE $\left.=0.05, \quad p<0.001, \quad \eta_{p}^{2}=0.17\right)$, confirming the results from the Friedman test on the binned data presented above. The effects of sleep stage, however, and the interaction of sleep stage with time SSO were not significant $\left(F(1,122)=0.001, \mathrm{MSE}=0.05, p=0.976, \eta_{p}^{2}=0.00\right.$ and $F(1,122)=0.02, \quad \mathrm{MSE}=0.05, \quad p=0.884, \quad \eta_{p}^{2}=0.00$, respectively).

\section{Discussion}

Comparison of OT and CT assay levels with previous literature

For CT levels, our assays were very close to previous findings. For example, Papanicolaou et al. (2002) found salivary CT values of approximately $0.3 \mu \mathrm{g} / \mathrm{dl}$ at 2330 hours and $0.75 \mu \mathrm{g} / \mathrm{dl}$ at 0730 hours. For OT, our assays were somewhat higher than, but in accord with, previous findings, although it should be noted that those previous findings do not, in general, have samples taken solely from the late night and early morning sampling times that were included here. Carter et al. (2007) report OT levels of 4-10 pg/ml in one study involving females, and $2 \mathrm{pg} / \mathrm{ml}$ in a study of males. White-Traut et al. (2009) found that salivary OT values for females ranged from 6.44 to $61.05 \mathrm{pg} / \mathrm{ml}$, with the highest mean for before breastfeeding of $38.30 \mathrm{pg} / \mathrm{ml}$, falling to the lowest level at the initiation of feeding $(16.58 \mathrm{pg} / \mathrm{ml})$.

\section{OT/CT and sleep}

OT levels did not change across the night, in accordance with Amico et al. (1983) and with the normal control group in Parker et al. (2010). There was no difference in OT levels between REM and N2 sleep when time SSO was controlled. In accordance with a large previous literature on the circadian rhythm of CT secretion (e.g., reviewed in Pannain and Van Cauter 2007; Kalsbeek et al. 2012), CT significantly increased during the second half of the sleep period. There was no difference in CT levels between REM and N2 sleep when time SSO was controlled.

An important implication of the present study concerns the neurobiology of memory consolidation during sleep. REM sleep and the N2 and N3 stages of non-REM sleep have been associated with memory consolidation in many 
Table 1 Wilcoxon test statistical significances for differences in cortisol levels between sampling periods shown in Fig. 4

\begin{tabular}{llllr}
\hline & $<4$ h SSO & $4-6.6$ h SSO & $>6.6$ h SSO & Post-sleep \\
\hline Pre-sleep & \multirow{2}{*}{ NS } & NS & NS & 0.001 \\
$<4$ h SSO & & NS & 0.001 & $<0.001$ \\
$4-6.6$ h SSO & & & $<0.005$ & $<0.001$ \\
$>6.6$ h SSO & & & & 0.001 \\
\hline
\end{tabular}

NS not significant

To correct for multiple comparisons, a threshold of $p=0.005$ is used as a criterion for significant difference

Table 2 Levels of salivary oxytocin $(\mathrm{pg} / \mathrm{ml})$ and cortisol $(\mu \mathrm{g} / \mathrm{dl})$ sampled after 10 min of REM or N2 sleep, from early $(<4 \mathrm{~h}$ since sleep onset), middle (4-6.6 h SSO) and late ( $>6.6$ h SOO) sleep

\begin{tabular}{|c|c|c|c|c|c|}
\hline \multirow[t]{2}{*}{ Period } & \multirow[t]{2}{*}{$n$} & \multicolumn{2}{|l|}{ REM } & \multicolumn{2}{|l|}{$\mathrm{N} 2$} \\
\hline & & Mean & SD & Mean & SD \\
\hline \multicolumn{6}{|c|}{ Oxytocin (pg/ml) } \\
\hline Early & 8 & 10.56 & 4.30 & 14.57 & 10.42 \\
\hline Middle & 7 & 13.81 & 5.82 & 14.39 & 9.46 \\
\hline Late & 6 & 10.60 & 4.26 & 12.14 & 7.77 \\
\hline \multicolumn{6}{|c|}{ Cortisol $(\mu \mathrm{g} / \mathrm{dl})$} \\
\hline Early & 7 & 0.18 & 0.14 & 0.23 & 0.28 \\
\hline Middle & 9 & 0.30 & 0.25 & 0.31 & 0.11 \\
\hline Late & 9 & 0.36 & 0.17 & 0.50 & 0.26 \\
\hline
\end{tabular}

recent papers (for reviews see Walker and Stickgold 2006; Diekelmann et al. 2009; Diekelmann and Born 2010; Smith 2010). However, study of the differential contribution of these stages of sleep to the various types of memory is confounded by the changing levels of CT in the night, as $\mathrm{CT}$ increases at the time of night that REM sleep becomes predominant. Furthermore, CT not only can impede memory consolidation and retrieval (Payne and Nadel 2004; Born and Wagner 2004, 2007; Payne 2010) but can also enhance encoding and consolidation of emotionally salient information (de Quervain et al. 2009). This study is relevant to proposals for mnemonic consolidation functions of REM sleep and of $\mathrm{N} 2$, in that it indicates that during the later part of sleep CT is as high in N2 as it is in REM. If REM memory-consolidation processes late in sleep are subject to increased CT levels at that time, as proposed by Payne and Nadel (2004), Wagner and Born (2008) and Payne (2010), then N2 memory-consolidation mechanisms (Peters et al. 2008; Fogel and Smith 2010) may be subject to the same cortisol-induced processes. Cortisol can have a disruptive effect on learning and memory (e.g. Born and Wagner 2007; Pace-Scott et al. 2012), and this may be a part of its contribution to longer term REM sleep-dependent memory consolidation (Payne et al. 2009). Born and Wagner (2007) note that early-night sleep, where SWS (N3) predominates, has low cortisol. There is thus the question regarding N2 sleep, which is evenly distributed across the night, of whether cortisol is involved in memory consolidation in late sleep N2.

For OT, the method and results here indicate the feasibility of using saliva sampling as a tool in the social neuroscience of attachment, such as by comparing nighttime OT in single versus co-sleeping individuals. In such cases, this study provides a normal baseline against which such findings might be compared. A beneficial translational by-product of looking at OT and sleep is that it may reveal underlying neurohormonal mechanisms for REMrelated erections. OT is required for erection and orgasm (Andersson 2001; Hirshkowitz and Schmidt 2005), and it may be involved in REM-related erections. Since sleeprelated erections are monitored for diagnostic purposes in cases of sexual dysfunction, the assessment of OT during sleep may be relevant to those issues as well. The results of the present study show that saliva sampling for OT may be a feasible method to be used in the home environment for these medical investigations.

\section{Limitations to the study}

There are three limitations to the present study. Firstly, the saliva-sampling technique obviously involved waking participants, and this will have caused some disruption to their sleep cycles, and for some participants, could have been unpleasant or even stressful. Thus, there is a possibility that the multiple awakenings could have affected the levels of OT, and, in particular, CT. Spath-Schwalbe et al. (1991) indeed did find that arousal from the beginning of the second REM sleep period, which is close to the first significant nocturnal rise in plasma cortisol, led to a significant increase in plasma cortisol for the period immediately after the arousal compared with undisturbed sleep on baseline nights. However, Hucklebridge et al. (2000) found no significant difference in salivary cortisol between spontaneous morning awakening and morning awakening caused by deliberate interruption of sleep. Furthermore, both these morning awakening cortisol levels were significantly higher than for cortisol after an interruption of sleep in the middle of the night (at $4 \mathrm{~h}$ prior to normal awakening time), and nocturnal interruption of sleep did not affect the subsequent morning response. We accept that there was a limitation to the current study due to the multiple awakenings design, however, it should also be noted that the alternative of serum sampling can itself affect sleep quality (Vitiello et al. 1996). Future work should assess subjective sleep quality, as this may affect CT levels during the night, and affect the cortisol awakening response (Backhaus et al. 2004). 
The second limitation is that saliva was not sampled during N3. The third limitation is that saliva and serum assays are measures of peripheral rather than CNS hormones, and for OT, it is unclear whether peripheral levels are associated with CNS neuropeptide levels, although there may be some coordination between them (Gimpl and Fahrenholz 2001; Carter et al. 2007). The fact that OT secreted into the peripheral circulation from the posterior pituitary does not cross the blood-brain barrier makes the precise relationships between peripheral levels and centrally mediated behaviour unclear and controversial (Gimpl and Fahrenholz 2001; Ross and Young 2009; MeyerLindenberg et al. 2011). As an example relevant to the current study, a midday peak in OT levels is seen in human CSF but not peripheral circulation (Amico et al. 1983). Nonetheless, oxytocinergic neurons in the PVN and supraoptic (SON) hypothalamic nuclei (Landgraf and Neumann 2004) project widely to limbic structures in both the rodent and human brain (Gimpl and Fahrenholz 2001; Ross and Young 2009). Ross and Young (2009) offer a possible explanation for this apparent paradox in that the dendrites, soma and unmyelinated axon collaterals of oxytocinergic neurons can release OT, independently of action potentials, which then can diffuse into surrounding limbic regions. As the specificity of neuropeptide action is determined largely by receptor type (Ludwig and Leng 2006), such broad release can remain restricted to particular targets (Ross and Young 2009). Because many oxytocinergic fibers in limbic areas originate in magnocellular neurons of the PVN and anterior SON projecting to the posterior pituitary, non-synaptic OT release in key limbic structures such as the nucleus accumbens can occur in concert with its release into peripheral circulation (Ross and Young 2009). The constancy we have found for salivary OT across the night, and the lack of difference in salivary OT between REM and N2 sleep, therefore, may well reflect similar characteristics in central OT.

Aside from these three main limitations, there may be some limitation resulting from our use of students for the experiment. However, the students we used had been screened so as to exclude those with extremely variable sleep habits. In addition, the experiment ran during a term in which no examinations were held, so as to exclude the possibility of stress due to examinations.

\section{Conclusions}

Levels of oxytocin did not change significantly during the night. There were no significant differences in levels of oxytocin or cortisol between REM and N2 awakenings when time SSO was controlled. The study has implications for theories of sleep-dependent memory consolidation that incorporate the increase in cortisol that occurs late in sleep, and for the medical assessment of oxytocin during sleep.

Acknowledgments Study was funded by the Wales Institute for Cognitive Neuroscience.

\section{References}

Amico JA, Tenicela R, Johnston J, Robinson AG (1983) A timedependent peak of oxytocin exists in cerebrospinal fluid but not in plasma of humans. J Clin Endocrinol Metab 57:947-951

Andersson K-E (2001) Pharmacology of penile erection. Pharmacol Rev 53:417-450

Backhaus J, Junghanns K, Hohagen F (2004) Sleep disturbances are correlated with decreased morning awakening salivary cortisol. Psychoneuroendocrinology 29:1184-1191. doi:10.1016/j.psyneuen. 2004.01.010

Bartz JA, Hollander E (2006) The neuroscience of affiliation: forging links between basic and clinical research on neuropeptides and social behaviour. Horm Behav 50:518-528. doi:10.1016/j.yhbeh. 2006.06.018

Bierwolf C, Struve K, Marshall L, Born J, Fehm HL (1997) Slow wave sleep drives inhibition of Pituitary-Adrenal secretion in humans. J Neuroendocrin 9:479-484

Bonnet MH, Arand DL (2010) Hyperarousal and insomnia: state of the science. Sleep Med Rev 14:9-15. doi:10.1016/j.smrv.2009. 05.002

Born J, Wagner U (2004) Memory consolidation during sleep: role of cortisol feedback. Ann NY Acad Sci 1032:198-201. doi:10.1196/ annals. 1314.020

Born J, Wagner U (2007) Sleep, hormones, and memory. Sleep Med Clin 2:209-227. doi:10.1016/j.jsmc.2007.03.005

Born J, Kern W, Bieber K, Fehm-Wolfsdorf G, Schiebe M, Fehm HL (1986) Night time plasma cortisol secretion is associated with specific sleep stages. Biol Psychiat 21:1415-1424. doi:10.1016/ 0006-3223(86)90333-1

Campbell A (2010) Oxytocin and human social behavior. Pers Soc Psychol Rev 14:281-295. doi:10.1177/1088868310363594

Carter CS, Pournajafi-Nazarloo H, Kramer KM, Ziegler TE, WhiteTraut R, Bello D, Schwertz D (2007) Oxytocin behavioral associations and potential as a salivary biomarker. Ann NY Acad Sci 1098:312-322. doi:10.1196/annals.1384.006

Davidson JR, Moldofsky H, Lue FA (1991) Growth hormone and cortisol secretion in relation to sleep and wakefulness. J Psychiat Neurosci 16:96-102

de Quervain DJ, Aerni A, Schelling G, Roozendaal B (2009) Glucocorticoids and the regulation of memory in health and disease. Front Neuroendocrin 30:358-370. doi:10.1016/j.yfrne.2009.03.002

Diekelmann S, Born J (2010) The memory function of sleep. Nat Rev Neurosci 11:114-126. doi:10.1038/nrn2762

Diekelmann S, Wilhelm I, Born J (2009) The whats and whens of sleep-dependent memory consolidation. Sleep Med Rev 13:309321. doi:10.1016/j.smrv.2008.08.002

Elder GJ, Ellis JG, Wetherell MA (2012) Associations between stages of sleep and the cortisol awakening response. Sleep 35(Abstract supplement):A56

Fogel SM, Smith CT (2010) The function of the sleep spindle: a physiological index of intelligence and a mechanism for sleepdependent memory consolidation. Neurosci Biobehav Rev 35:1154 1165. doi:10.1016/j.neubiorev.2010.12.003

Follenius M, Brandenberger G, Simon C, Schlienger JL (1988) REM sleep in humans begins during decreased secretory activity of the anterior pituitary. Sleep 11:546-555 
Follenius M, Brandenberger G, Bandesapt JJ, Libert JP, Ehrhart J (1992) Nocturnal cortisol release in relation to sleep structure. Sleep 15:21-27

Forsling ML (1993) Neurohypophysial hormones and circadian rhythm. Ann NY Acad Sci 689:382-395

Forsling ML, Montgomery H, Halpin D, Windle RJ, Treacher DF (1998) Daily patterns of secretion of neurohypophysial hormones in man: effect of age. Exp Physiol 83:409-418

Gais S, Born J (2004) Declarative memory consolidation: mechanisms acting during human sleep. Learn Mem 11:679-685. doi: 10.1101/lm.80504

Gimpl G, Fahrenholz F (2001) The oxytocin receptor system: structure, function, and regulation. Physiol Rev 81:629-683

Goldberg DP, Williams P (1991) A user's guide to the General Health Questionnaire. NFER-Nelson Publishing Company Ltd., Windsor

Gordis EB, Granger DA, Susman EJ, Trickett PK (2006) Asymmetry between salivary cortisol and alpha-amylase reactivity to stress: relation to aggressive behavior in adolescents. Psychoneuroendocrinology 31:976-987. doi:10.1016/j.psyneuen.2006.05.010

Gordis EB, Granger DA, Susman EJ, Trickett PK (2008) Salivary alpha amylase-cortisol asymmetry in maltreated youth. Horm Behav 53:96-103. doi:10.1016/j.yhbeh.2007.09.002

Gordon I, Martin C, Feldman R, Leckman JF (2011) Oxytocin and social motivation. Dev Cogn Neurosci 1:471-493. doi:10.1016/ j.den.2011.07.007

Grewen KM, Davenport RE, Light KC (2010) An investigation of plasma and salivary oxytocin responses in breast- and formulafeeding mothers of infants. Psychophysiology 47:625-632. doi: 10.1111/j.1469-8986.2009.00968.x

Gronfier C, Brandenberger G (1998) Ultradian rhythms in pituitary and adrenal hormones: their relations to sleep. Sleep Med Rev 2:17-29

Gronfier C, Luthringer R, Follenius M, Schaltenbrand N, Macher JP, Muzet A, Brandenberger G (1997) Temporal relationships between pulsatile cortisol secretion and electroencephalographic activity during sleep in man. Electroenceph Clin Neurophysiol 103:405-408

Gronfier C, Simon C, Piquard F, Ehrhart J, Brandenberger G (1999) Neuroendocrine processes underlying ultradian sleep regulation in man. J Clin Endocrinol Metab 84:2686-2690. doi:10.1210/ jc.84.8.2686

Heinrichs M, Von Dawans B, Domes G (2009) Oxytocin, vasopressin, and human social behavior. Front Neuroendocrin 30:548-557. doi:10.1016/j.yfrne.2009.05.005

Hirshkowitz M, Schmidt MH (2005) Sleep-related erections: clinical perspectives and neural mechanisms. Sleep Med Rev 9:311-329. doi:10.1016/j.smrv.2005.03.001

Hollander E, Novotny S, Hanratty M, Yaffe R, DeCaria CM, Aronowitz BR, Mosovich S (2003) Oxytocin infusion reduces repetitive behaviors in adults with autistic and Asperger's disorders. Neuropsychopharmacology 28:193-198. doi:10.1038/ sj.npp. 1300021

Horne JA (2000) REM sleep: by default? Neurosci Biobehav Rev 24:777-797. doi:10.1016/S0149-7634(00)00037-3

Hucklebridge F, Clow A, Rahman H, Evans P (2000) The cortisol response to normal and nocturnal awakening. J Psychophysiol 14:24-28. doi:10.1027//0269-8803.14.1.24

Iber C, Ancoli-Israel S, Chesson A, Quan SF, For the American Academy of Sleep Medicine (2007) The AASM manual for the scoring of sleep and associated events: rules, terminology and technical specifications, 1st edn. American Academy of Sleep Medicine, Winchester

Kalsbeek A, van der Spek R, Lei J, Endert E, Buijs RM, Fliers E (2012) Circadian rhythms in the hypothalamo-pituitary-adrenal
(HPA) axis. Mol Cell Endocrinol 349:20-29. doi: 10.1016/j.mce.2011.06.042

Lancel M, Krömer S, Neumann ID (2003) Intracerebral oxytocin modulates sleep-wake behaviour in male rats. Regul Peptides 114:145-152

Landgraf R, Neumann ID (2004) Vasopressin and oxytocin release within the brain: a dynamic concept of multiple and variable modes of neuropeptide communication. Frontiers Neuroendocrin 25:150-176. doi:10.1016/j.yfrne.2004.05.001

Lorr M (1984) Profile of Mood States: bi-polar form (POMS-BI): manual. Educational and Industrial Testing Service, San Diego

Ludwig M, Leng G (2006) Dendritic peptide release and peptide-dependent behaviours. Nat Rev Neurosci 7:126-136. doi:10.1038/nrn1845

McNamara P, Andresen J, Clark J, Zborowski M, Duffy C (2001) Impact of attachment styles on dream recall and dream content: a test of the attachment hypothesis of REM sleep. J Sleep Res 10:117-127. doi:10.1046/j.1365-2869.2001.00244.x

Meyer-Lindenberg A, Domes G, Kirsch P, Heinrichs M (2011) Oxytocin and vasopressin in the human brain: social neuropeptides for translational medicine. Nat Rev Neurosci 12:524-538. doi: $10.1038 / \mathrm{nrn} 3044$

Nielsen T, Lara-Carrasco J (2007) Nightmares, dreaming, and emotion regulation: a review. In: Barrett D, McNamara P (eds) The new science of dreaming, vol 2. Praeger, London, pp 253-284

Pace-Scott EF, Vijayakumar S, Milad MR, Orr SP, Pitman RK, Spencer R (2012) Endogenous cortisol levels predict poorer extinction learning in the morning but not the evening. Sleep 35(Abstract supplement):A99

Panksepp J (2006) Emotional endophenotypes in evolutionary psychiatry. Prog Neuropsychopharmacol Biol Psychiatry 30:774-784. doi:10.1016/j.pnpbp.2006.01.004

Pannain S, Van Cauter E (2007) Modulation of endocrine function by sleep-wake homeostasis and circadian rhythmicity. Sleep Med Clin 2:147-159. doi:10.1016/j.jsmc.2007.03.001

Papanicolaou DA, Mullen N, Kyrou I, Nieman LK (2002) Nighttime salivary cortisol: a useful test for the diagnosis of Cushing's Syndrome. J Clin Endocrinol Metab 87:4515-4521. doi: 10.1210/jc.2002-020534

Parker KJ, Kenna HA, Zeitzer JM, Keller J, Blasey CM, Amico JA, Schatzberg AF (2010) Preliminary evidence that plasma oxytocin levels are elevated in major depression. Psychiat Res 178:359-362. doi:10.1016/j.psychres.2009.09.017

Payne JD (2010) Memory consolidation, the diurnal rhythm of cortisol, and the nature of dreams: a new hypothesis. Int Rev Neurobiol 92:101-134. doi:10.1016/S0074-7742(10)92006-0

Payne JD, Nadel L (2004) Sleep, dreams, and memory consolidation: the role of the stress hormone cortisol. Learn Mem 11:671-678. doi:10.1101/lm.77104

Payne JD, Schacter DL, Propper R, Huang L-W, Wamsley E, Tucker MA, Walker MP, Stickgold R (2009) The role of sleep in false memory formation. Neurobiol Learn Mem 92:327-334. doi: 10.1016/j.nlm.2009.03.007

Peters KR, Ray LB, Smith V, Smith CT (2008) Changes in the density of stage 2 sleep spindles following motor learning in young and older adults. J Sleep Res 17:23-33. doi:10.1111/j.1365-2869. 2008.00634.x

Ross HE, Young LJ (2009) Oxytocin and the neural mechanisms regulating social cognition and affiliative behavior. Front Neuroendocrinol 30:534-547. doi:10.1016/j.yfrne.2009.05.004

Smith C (2010) Sleep states and memory processes in humans: procedural versus declarative memory systems. Sleep Med Rev 5:491-506. doi:10.1053/smrv.2001.0164

Spath-Schwalbe E, Gofferje M, Kern W, Born J, Fehm HL (1991) Sleep disruption alters nocturnal ACTH and cortisol secretory patterns. Biol Psychiatry 29:575-584 
Tomfohr LM, Edwards KM, Dimsdale JE (2012) Is obstructive sleep apnea associated with cortisol levels? A systematic review of the research evidence. Sleep Med Rev 16:243-249. doi:10.1016/j.smrv. 2011.05.003

Troxel WM (2010) It's more than sex: exploring the dyadic nature of sleep and implications for health. Psychosom Med 72:578-586. doi:10.1097/PSY.0b013e3181de7ff8

Ulrich-Lai YM, Herman JP (2009) Neural regulation of endocrine and autonomic stress responses. Nat Rev Neurosci 10:397-409. doi: $10.1038 / \mathrm{nrn} 2647$

Van Cauter E, Tasali E (2011) Endocrine physiology in relation to sleep and sleep disturbances. In: Kryger MH, Roth T, Dement WC (eds) Principles and practice of sleep medicine, 5th edn. Elsevier, Philadelphia, pp 291-311

Vitiello MV, Larsen LH, Moe KE, Borson S, Schwartz RS, Prinz PN (1996) Objective sleep quality of healthy older men and women is differentially disrupted by nighttime periodic blood sampling via indwelling catheter. Sleep 19:304-311
Wagner U, Born J (2008) Memory consolidation during sleep: interactive effects of sleep stages and HPA regulation. Stress 11:28-41. doi:10.1080/10253890701408822

Wagner U, Gais S, Born J (2001) Emotional memory formation is enhanced across sleep intervals with high amounts of rapid eye movement sleep. Learn Mem 8:112-119. doi:10.1101/lm.36801

Walker MP, Stickgold R (2006) Sleep, memory, and plasticity. Annu Rev Psychol 57:139-166. doi:10.1146/annurev.psych.56.091103. 070307

White-Traut R, Watanabe K, Pournajafi-Nazarloo H, Schwertz D, Bell A, Carter CS (2009) Detection of salivary oxytocin levels in lactating women. Dev Psychobiol 51:367-373. doi:10.1002/dev. 20376

Zetzsche T, Frasch A, Jirikowski G, Murek H, Steiger A (1996) Nocturnal oxytocin secretion is reduced in major depression. Biol Psychiatry 39:584. doi:10.1016/0006-3223(96)84235-1 